\title{
GROWTH ESTIMATES FOR THE MAXIMAL TERM AND CENTRAL EXPONENT OF THE DERIVATIVE OF A DIRICHLET SERIES
}

\author{
FEDYNYAK S.I. ${ }^{1}$, FILEVYCH P.V. ${ }^{2}$
}

Let $A \in(-\infty,+\infty], \Phi:[a, A) \rightarrow \mathbb{R}$ be a continuous function such that $x \sigma-\Phi(\sigma) \rightarrow-\infty$ as $\sigma \uparrow A$ for every $x \in \mathbb{R}, \widetilde{\Phi}(x)=\max \{x \sigma-\Phi(\sigma): \sigma \in[a, A)\}$ be the Young-conjugate function of $\Phi, \bar{\Phi}(x)=\widetilde{\Phi}(x) / x$ and $\Gamma(x)=(\widetilde{\Phi}(x)-\ln x) / x$ for all sufficiently large $x,\left(\lambda_{n}\right)$ be a nonnegative sequence increasing to $+\infty$, and $F(s)=\sum_{n=0}^{\infty} a_{n} e^{s \lambda_{n}}$ be a Dirichlet series such that its maximal term $\mu(\sigma, F)=\max \left\{\left|a_{n}\right| e^{\sigma \lambda_{n}}: n \geq 0\right\}$ and central index $v(\sigma, F)=\max \left\{n \geq 0:\left|a_{n}\right| e^{\sigma \lambda_{n}}=\mu(\sigma, F)\right\}$ are defined for all $\sigma<A$. It is proved that if $\ln \mu(\sigma, F) \leq(1+o(1)) \Phi(\sigma)$ as $\sigma \uparrow A$, then the inequalities

$$
\varlimsup_{\sigma \uparrow A} \frac{\mu\left(\sigma, F^{\prime}\right)}{\mu(\sigma, F) \bar{\Phi}^{-1}(\sigma)} \leq 1, \quad \varlimsup_{\sigma \uparrow A} \frac{\lambda_{v\left(\sigma, F^{\prime}\right)}}{\Gamma^{-1}(\sigma)} \leq 1,
$$

hold, and these inequalities are sharp.

Key words and phrases: Dirichlet series, maximal term, central index, central exponent, Youngconjugate function.

${ }^{1}$ Ukrainian Catholic University, 2A Kozelnytska str., 79076, Lviv, Ukraine

2 Lviv Polytechnic National University, 5 Mytropolyt Andrei str., 79013, Lviv, Ukraine

E-mail: napets.fed@gmail.com (Fedynyak S.I.), p.v.filevych@gmail.com (Filevych P.V.)

\section{INTRODUCTION} form

We fix a nonnegative sequence $\left(\lambda_{n}\right)$ increasing to $+\infty$, and consider a Dirichlet series of the

$$
F(s)=\sum_{n=0}^{\infty} a_{n} e^{s \lambda_{n}}
$$

For this series, by $\sigma_{a}(F)$ we denote its abscissa of absolute convergence. Put

$$
\beta(F)=\varliminf_{n \rightarrow \infty} \frac{1}{\lambda_{n}} \ln \frac{1}{\left|a_{n}\right|},
$$

and let

$$
\begin{aligned}
& E_{1}(F)=\left\{\sigma \in \mathbb{R}:\left|a_{n}\right| e^{\sigma \lambda_{n}}=o(1), n \rightarrow \infty\right\}, \\
& E_{2}(F)=\left\{\sigma \in \mathbb{R}:\left|a_{n}\right| e^{\sigma \lambda_{n}}=O(1), n \rightarrow \infty\right\} .
\end{aligned}
$$

It is easy to see that for $j=1,2$ we have

$$
\beta(F)=\left\{\begin{array}{lll}
-\infty, & \text { if } & E_{j}(F)=\varnothing, \\
\sup E_{j}(F), & \text { if } & E_{j}(F) \neq \varnothing,
\end{array}\right.
$$

$\mathrm{y} \Delta \mathrm{K} 517.53$

2010 Mathematics Subject Classification: 30B50. 
i.e., the interval $(-\infty, \beta(F))$ is the domain of existence for the maximal term

$$
\mu(\sigma, F)=\max \left\{\left|a_{n}\right| e^{\sigma \lambda_{n}}: n \geq 0\right\}
$$

of series (1). Since $\beta\left(F^{\prime}\right)=\beta(F)$, this interval is also the domain of existence for the maximal term of the derivative of series (1).

It is well known (for instance, see [8, pp. 114-115]) that for every Dirichlet series of the form (1) we have

$$
\sigma_{a}(F) \leq \beta(F) \leq \sigma_{a}(F)+\tau, \quad \tau:=\varlimsup_{n \rightarrow \infty} \frac{\ln n}{\lambda_{n}},
$$

and these inequalities are sharp. Moreover, it was shown in [4] that for any $A, B \in[-\infty,+\infty]$ such that $A \leq B \leq A+\tau$ there exists a Dirichlet series of the form (1) for which $\sigma_{a}(F)=A$ and $\beta(F)=B$.

We assume that every Dirichlet series of the form (1) considered below is not reduced to a constant, that is, for this series we have $a_{n} \lambda_{n} \neq 0$ for at least one integer $n \geq 0$. By this assumption, the central index

$$
v(\sigma, F)=\max \left\{n \geq 0:\left|a_{n}\right| e^{\sigma \lambda_{n}}=\mu(\sigma, F)\right\}
$$

of series (1) and the central index of the derivative of this series are defined for all $\sigma<\beta(F)$.

Let $A \in(-\infty,+\infty]$, and $\Phi: D_{\Phi} \rightarrow \mathbb{R}$ be a real function. We say that $\Phi \in \Omega_{A}$ if the domain $D_{\Phi}$ of $\Phi$ is an interval of the form $[a, A), \Phi$ is continuous on $D_{\Phi}$, and the following condition

$$
\forall x \in \mathbb{R}: \quad \lim _{\sigma \uparrow A}(x \sigma-\Phi(\sigma))=-\infty
$$

holds. It is easy to see that in the case $A<+\infty$ condition (4) is equivalent to the condition $\Phi(\sigma) \rightarrow+\infty, \sigma \rightarrow A-0$, and in the case $A=+\infty$ this condition is equivalent to the condition $\Phi(\sigma) / \sigma \rightarrow+\infty, \sigma \rightarrow+\infty$. For $\Phi \in \Omega_{A}$ by $\widetilde{\Phi}$ we denote the Young-conjugate function of $\Phi$, i.e.,

$$
\widetilde{\Phi}(x)=\max \left\{x \sigma-\Phi(\sigma): \sigma \in D_{\Phi}\right\}, \quad x \in \mathbb{R} .
$$

Note (see Lemma 1 below), that the function $\bar{\Phi}(x)=\widetilde{\Phi}(x) / x$ is continuous and increasing to $A$ on some interval of the form $\left(x_{0},+\infty\right)$. Hence the inverse function $\bar{\Phi}^{-1}$ is defined on some interval of the form $\left(A_{0}, A\right)$ and $\bar{\Phi}^{-1}$ is continuous and increasing to $+\infty$ on $\left(A_{0}, A\right)$.

We say that $\Phi \in \Omega_{A}^{\prime}$, if $\Phi \in \Omega_{A}, \Phi$ is continuously differentiable on $D_{\Phi}$, and $\Phi^{\prime}$ is positive and increasing on $D_{\Phi}$.

Let $\Phi \in \Omega_{A}^{\prime}$. It is clear that $\Phi^{\prime}(\sigma) \uparrow+\infty$ as $\sigma \uparrow A$. In addition, $\Phi^{\prime}$ has an inverse function $\varphi:\left[x_{0},+\infty\right) \rightarrow D_{\Phi}$. Set

$$
\widehat{\Phi}(\sigma)=\sigma-\frac{\Phi(\sigma)}{\Phi^{\prime}(\sigma)}, \quad \sigma \in D_{\Phi}
$$

It is easy to prove that $\bar{\Phi}(x)=\widehat{\Phi}(\varphi(x))$ for every $x \in\left(x_{0},+\infty\right)$. This implies that $\Phi^{\prime}\left(\widehat{\Phi}^{-1}(\sigma)\right)=\bar{\Phi}^{-1}(\sigma)$ for all $\sigma \in\left(A_{0}, A\right)$.

M.M. Sheremeta [9] proved the following two theorems.

Theorem A. Suppose that $A \in(-\infty,+\infty], \Phi \in \Omega_{A}^{\prime}$, and the condition

$$
\ln \Phi^{\prime}(\sigma)=o(\Phi(\sigma)), \quad \sigma \uparrow A,
$$


holds. Then for every Dirichlet series of the form (1) such that $\sigma_{a}(F)=A$ and

$$
\varlimsup_{\sigma \uparrow A} \frac{\ln \mu(\sigma, F)}{\Phi(\sigma)}=1
$$

we have

$$
\varlimsup_{\sigma \uparrow A} \frac{\mu\left(\sigma, F^{\prime}\right)}{\mu(\sigma, F) \bar{\Phi}^{-1}(\sigma)} \leq 1
$$

Theorem B. Suppose that $A \in(-\infty,+\infty], \Phi \in \Omega_{A^{\prime}}^{\prime}$ there exists a number $\alpha \in(0,1]$ such that the function $h(\sigma)=\left(\Phi^{\prime}(\sigma)\right)^{\alpha} / \Phi(\sigma)$ is nonincreasing on $\left[\sigma_{0}, A\right)$, and $\lambda_{n}=o\left(\lambda_{n+1}\right)$ as $n \rightarrow+\infty$. If

$$
F(s)=\sum_{n=0}^{\infty} e^{-\widetilde{\Phi}\left(\lambda_{n}\right)} e^{s \lambda_{n}},
$$

then

$$
\varlimsup_{\sigma \uparrow A} \frac{\mu\left(\sigma, F^{\prime}\right)}{\mu(\sigma, F) \bar{\Phi}^{-1}(\sigma)}=1
$$

Remark 1. Clearly, if for a Dirichlet series of the form (1) with $\sigma_{a}(F)=A$ equality (6) holds, then for this series we have $\beta(F)=A$.

Remark 2. It can be proved that for series (8) by the conditions of Theorem B relation (6) holds (this is also clear from considerations given in [9]).

Remark 3. In the proofs of Theorems A and B suggested in [9], the obvious inequalities

$$
\lambda_{v(\sigma, F)} \leq \frac{\mu\left(\sigma, F^{\prime}\right)}{\mu(\sigma, F)} \leq \lambda_{v\left(\sigma, F^{\prime}\right)}, \quad \sigma<\beta(F)
$$

were used, and, in fact, the following more exactly results were proved: by the conditions of Theorem A for every Dirichlet series of the form (1) the inequality

$$
\varlimsup_{\sigma \uparrow A} \frac{\lambda_{v\left(\sigma, F^{\prime}\right)}}{\bar{\Phi}^{-1}(\sigma)} \leq 1
$$

holds, and by the conditions of Theorem B for series (8) we have

$$
\varlimsup_{\sigma \uparrow A} \frac{\lambda_{v(\sigma, F)}}{\bar{\Phi}^{-1}(\sigma)}=1 .
$$

Therefore, for every Dirichlet series of the form (1) with $\sigma_{a}(F)=A$, by some conditions on a function $\Phi \in \Omega_{A}^{\prime}$, equality (6) implies estimates (7) and (11), and these estimates are sharp.

In [9], M.M. Sheremeta conjectured that in Theorem A condition (5) may be unnecessary, that is, Theorem $A$ is true without any additional condition on a function $\Phi \in \Omega_{A}^{\prime}$. Below we confirm this conjecture. Moreover, we prove that inequality (7) is sharp in the case of an arbitrary function $\Phi \in \Omega_{A}^{\prime}$. In addition, in the case of an arbitrary $\Phi \in \Omega_{A}^{\prime}$ we obtain a sharp growth estimate for the central exponent $\lambda_{v\left(\sigma, F^{\prime}\right)}$ of the derivative of a Dirichlet series, which, generally, does not coincide with estimate (11). 


\section{MAIN RESULTS}

Let $A \in(-\infty,+\infty]$. For a Dirichlet series of the form (1) with $\beta(F)=A$ and a function $\Phi \in \Omega_{A}$ we put

$$
t_{\Phi}(F)=\varlimsup_{\sigma \uparrow A} \frac{\ln \mu(\sigma, F)}{\Phi(\sigma)} .
$$

Setting $\bar{\Phi}^{-1}(\sigma)=+\infty$ for all $\sigma \in[A,+\infty]$, we have

$$
t_{\Phi}(F)=\varlimsup_{n \rightarrow \infty} \frac{\lambda_{n}}{\bar{\Phi}-1\left(\frac{1}{\lambda_{n}} \ln \frac{1}{\left|a_{n}\right|}\right)}
$$

(see [7] and also [5]).

The following theorem confirms the above conjecture of M.M. Sheremeta.

Theorem 1. Let $A \in(-\infty,+\infty], \Phi \in \Omega_{A}$.

(i) For every Dirichlet series of the form (1) with $\beta(F)=A$ and $t_{\Phi}(F) \leq 1$ we have (7).

(ii) There exists a Dirichlet series of the form (1) with $\beta(F)=A$ and $t_{\Phi}(F)=1$ such that equality (9) holds.

Let $\Phi \in \Omega_{A}$. Since $\bar{\Phi}$ is continuous and increasing to $A$ on some interval of the form $\left(x_{0},+\infty\right)$, there exists $\alpha>e$ such that the function

$$
\Gamma(x)=\bar{\Phi}(x)-\frac{\ln x}{x}, \quad x \in[\alpha,+\infty),
$$

is continuous and increasing to $A$. Hence the inverse function $\Gamma^{-1}$ is defined on some interval of the form $\left[A_{1}, A\right)$ and $\Gamma^{-1}$ is continuous and increasing to $+\infty$ on $\left[A_{1}, A\right)$.

Theorem 2. Suppose that $A \in(-\infty,+\infty], \Phi \in \Omega_{A}$, and $\Gamma$ is defined by (14).

(i) For every Dirichlet series of the form (1) with $\beta(F)=A$ and $t_{\Phi}(F) \leq 1$ we have

$$
\varlimsup_{\sigma \uparrow A} \frac{\lambda_{v\left(\sigma, F^{\prime}\right)}}{\Gamma^{-1}(\sigma)} \leq 1
$$

(ii) There exists a Dirichlet series of the form (1) with $\beta(F)=A$ and $t_{\Phi}(F)=1$ such that

$$
\varlimsup_{\sigma \uparrow A} \frac{\lambda_{v\left(\sigma, F^{\prime}\right)}}{\Gamma^{-1}(\sigma)}=1
$$

Using Theorem 2, we show that without additional conditions on a function $\Phi \in \Omega_{A}^{\prime}$ estimate (11) may not be satisfied for some Dirichlet series of the form (1) with $\sigma_{a}(F)=A$ such that (6) holds. Indeed, let $\Phi(\sigma)=-\ln |\sigma|$ for all $\sigma \in[-1,0)$. It is easy to make sure that

$$
\bar{\Phi}^{-1}(\sigma) \sim \frac{1}{|\sigma|} \ln \frac{1}{|\sigma|}, \quad \Gamma^{-1}(\sigma) \sim \frac{2}{|\sigma|} \ln \frac{1}{|\sigma|} \quad \text { as } \sigma \uparrow A .
$$

By Theorem 2 there exists a Dirichlet series of the form (1) with $\beta(F)=0$ and $t_{\Phi}(F)=1$ such that equality (15) holds, that is

$$
\varlimsup_{\sigma \uparrow 0} \frac{\lambda_{v\left(\sigma, F^{\prime}\right)}}{\frac{1}{|\sigma|} \ln \frac{1}{|\sigma|}}=2 .
$$


Suppose that $\ln n=o\left(\lambda_{n}\right)$ as $n \rightarrow \infty$. Then by (3) we have $\sigma_{a}(F)=0$. Estimate (11) takes the form

$$
\varlimsup_{\sigma \uparrow 0} \frac{\lambda_{v\left(\sigma, F^{\prime}\right)}}{\frac{1}{|\sigma|} \ln \frac{1}{|\sigma|}} \leq 1
$$

and, obviously, this estimate is false.

Theorems 1 and 2 are consequences of the following two theorems.

Theorem 3. Let $A \in(-\infty,+\infty]$ and $\Phi \in \Omega_{A}$. For every Dirichlet series of the form (1) such that $\beta(F)=A$ and

$$
\ln \mu(\sigma, F) \leq \Phi(\sigma), \quad \sigma \in\left[\sigma_{1}, A\right)
$$

we have

$$
\frac{\mu\left(\sigma, F^{\prime}\right)}{\mu(\sigma, F)} \leq \bar{\Phi}^{-1}(\sigma), \quad \sigma \in\left[\sigma_{2}, A\right)
$$

Theorem 4. Let $A \in(-\infty,+\infty]$ and $\Phi \in \Omega_{A}$. There exists a Dirichlet series of the form (1) such that for an infinite set $E$ of positive integers we have

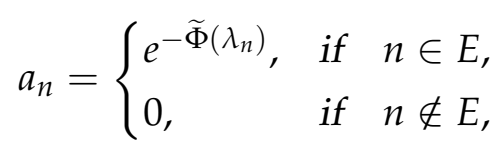

and this series satisfies (12).

Remark 4. Since for each $\Phi \in \Omega_{A}$ we have $\widetilde{\Phi}(x) / x=\bar{\Phi}(x) \rightarrow A$ as $x \rightarrow+\infty$, for a Dirichlet series of the form (1) whose existence follows from Theorem 4 we obtain $\beta(F)=A$ by (2).

Remark 5. If $\Phi \in \Omega_{A}$ and a Dirichlet series of the form (1) with $\beta(F)=A$ satisfies (16), then, by Theorem 3 and the left of inequalities (10), for all $\sigma \in\left[\sigma_{2}, A\right)$ we obtain $\lambda_{v(\sigma, F)} \leq \bar{\Phi}^{-1}(\sigma)$. Since $\lambda_{v(\sigma, F)}=(\ln \mu(\sigma, F))_{+}^{\prime}$ for every $\sigma<\beta(F)$, this fact is easy to prove without using Theorem 3 (see [2, Lemma 5] or [3, Lemma 4]).

In order to prove Theorems 1, 2, 3 and 4, we will need some auxiliary results, which are given in the next section.

\section{AUXILIARY RESUlTS}

The following lemma is well known (see, for example, [1, § 3.2], [7]).

Lemma 1. Suppose that $A \in(-\infty,+\infty], \Phi \in \Omega_{A}$, and, for all $x \in \mathbb{R}, \varphi(x)=\max \left\{\sigma \in D_{\Phi}\right.$ : $x \sigma-\Phi(\sigma)=\widetilde{\Phi}(x)\}$. Then the following statements are true:

(i) the function $\varphi$ is nondecreasing on $\mathbb{R}$;

(ii) the function $\varphi$ is continuous from the right on $\mathbb{R}$;

(iii) $\varphi(x) \rightarrow A, x \rightarrow+\infty$;

(iv) the right-hand derivative of $\widetilde{\Phi}(x)$ is equal to $\varphi(x)$ at every point $x \in \mathbb{R}$;

(v) if $x_{0}=\inf \{x>0: \Phi(\varphi(x))>0\}$, then the function $\Phi(x)=\widetilde{\Phi}(x) / x$ increases to $A$ on $\left(x_{0},+\infty\right)$ 
(vi) the function $\alpha(x)=\Phi(\varphi(x))$ is nondecreasing on $[0,+\infty)$.

In the following two lemmas, which are proved in [2], $\varphi$ and $x_{0}$ are defined by $\Phi$ in the same way as in Lemma 1.

Lemma 2. Let $A \in(-\infty,+\infty], \Phi \in \Omega_{A}, \sigma_{0}=\bar{\Phi}\left(x_{0}+0\right)$, and $\sigma \in\left(\sigma_{0}, A\right)$ be a fixed number. Then the minimum value of the function

$$
h(y)=\frac{\Phi(y)}{y-\sigma}, \quad y \in(\sigma, A),
$$

is $\bar{\Phi}^{-1}(\sigma)$ and this value is attained at the point $y=\varphi\left(\bar{\Phi}^{-1}(\sigma)\right)$.

Lemma 3. Let $\delta \in(0,1), A \in(-\infty,+\infty], \Phi \in \Omega_{A}, \sigma_{0}=\Phi\left(x_{0}+0\right)$, and $y(\sigma)=\varphi\left(\bar{\Phi}^{-1}(\sigma)\right)$ for all $\sigma \in\left(\sigma_{0}, A\right)$. Then

$$
\bar{\Phi}^{-1}\left(\sigma+\frac{\delta \Phi(y(\sigma))}{\bar{\Phi}^{-1}(\sigma)}\right) \leq \frac{\bar{\Phi}^{-1}(\sigma)}{1-\delta}, \quad \sigma \in\left(\sigma_{0}, A\right) .
$$

The following lemma is proved in [6].

Lemma 4. Let $A \in(-\infty,+\infty]$. If for a Dirichlet series of the form (1) there exists an increasing sequence $\left(n_{k}\right)_{k=0}^{\infty}$ of nonnegative integers such that $a_{n}=0$ for all $n<n_{0}, a_{n_{k}} \neq 0$ for every $k \geq 0$, and

$$
\varkappa_{k}:=\frac{\ln \left|a_{n_{k}}\right|-\ln \left|a_{n_{k+1}}\right|}{\lambda_{n_{k+1}}-\lambda_{n_{k}}} \uparrow A, \quad k \uparrow \infty, \quad\left|a_{n}\right| \leq\left|a_{n_{k}}\right| e^{\varkappa_{k}\left(\lambda_{n_{k}}-\lambda_{n}\right)}, \quad n \in\left(n_{k}, n_{k+1}\right), k \geq 0,
$$

then $\beta(F)=A$ and, in addition, $v(\sigma, F)=n_{0}$ for every $\sigma<\varkappa_{0}$ and $\nu(\sigma, F)=n_{k+1}$ for all $\sigma \in\left[\varkappa_{k}, \varkappa_{k+1}\right)$ and $k \geq 0$.

Lemma 5. Suppose that $h$ is a function increasing on $[\alpha, \beta), h(\alpha)=a, \lim _{x \uparrow \beta} h(x)=b$, and

$$
h^{-1}(\sigma):=\inf \{x \in[\alpha, \beta): h(x)>\sigma\}, \quad \sigma \in[a, b) .
$$

Then the following statements are true:

(i) $h^{-1}$ is nondecreasing continuous on $[a, b)$;

(ii) $h^{-1}(a)=\alpha, \lim _{\sigma \uparrow b} h^{-1}(\sigma)=\beta$;

(iii) $h(x+0)=\max \left\{\sigma \in[a, b): h^{-1}(\sigma) \leq x\right\}$ for each $x \in[\alpha, \beta)$.

Proof. Let

$$
E(\sigma)=\{x \in[\alpha, \beta): h(x)>\sigma\}, \quad \sigma \in[a, b) .
$$

If $\sigma_{1}, \sigma_{2} \in[a, b)$ and $\sigma_{1}<\sigma_{2}$, then $E\left(\sigma_{2}\right) \subset E\left(\sigma_{1}\right)$, and hence

$$
h^{-1}\left(\sigma_{1}\right)=\inf E\left(\sigma_{1}\right) \leq \inf E\left(\sigma_{2}\right)=h^{-1}\left(\sigma_{2}\right) .
$$

Therefore, $h^{-1}$ is nondecreasing on $[a, b)$.

If $x \in[\alpha, \beta)$ and $h(x)=\sigma$, then $h^{-1}(\sigma)=x$, i.e., the interval $[\alpha, \beta)$ is the range of $h^{-1}$. This and the monotonicity of the function $h^{-1}$ imply its continuity, as well as both equalities in (ii).

Let us prove (iii). Let $x_{0} \in[\alpha, \beta)$ and $\sigma_{0}=\max \left\{\sigma \in[a, b): h^{-1}(\sigma) \leq x_{0}\right\}$. Then $h^{-1}\left(\sigma_{0}\right)=x_{0}$. Therefore, if $x \in\left(x_{0}, \beta\right)$, then $h(x)>\sigma_{0}$, and hence $h\left(x_{0}+0\right) \geq \sigma_{0}$. Suppose that $h\left(x_{0}+0\right)=\sigma_{3}>\sigma_{0}$. Then $h(x)>\sigma_{3}$ for all $x \in\left(x_{0}, \beta\right)$, that is, $\left(x_{0}, \beta\right) \subset E\left(\sigma_{3}\right)$. Thus

$$
h^{-1}\left(\sigma_{3}\right)=\inf E\left(\sigma_{3}\right) \leq x_{0}
$$

This and the definition of $\sigma_{0}$ imply that $\sigma_{3} \leq \sigma_{0}$, which contradicts the assumption that $h\left(x_{0}+0\right)>\sigma_{0}$. Hence, $h\left(x_{0}+0\right)=\sigma_{0}$. 


\section{PROOF OF THEOREMS}

Proof of Theorem 3. Suppose that $A \in(-\infty,+\infty]$ and $\Phi \in \Omega_{A}$. Consider a Dirichlet series of the form (1) with $\beta(F)=A$ which satisfies (16).

Let $\sigma_{0}$ be defined as in Lemma 2, and $\varphi(x)=\widetilde{\Phi}_{+}^{\prime}(x)$ for all $x \in \mathbb{R}$. Condition (16) implies the existence of a number $\sigma_{2} \in\left(\sigma_{0}, A\right)$ such that

$$
\max \{1, \ln \mu(y, F)-\ln \mu(\sigma, F)\} \leq \Phi(y), \quad y, \sigma \in\left[\sigma_{2}, A\right) .
$$

By taking here $y=y(\sigma)$, where $y(\sigma)=\varphi\left(\bar{\Phi}^{-1}(\sigma)\right)$, and using Lemma 2 , we get

$$
\frac{\ln \mu(y(\sigma), F)-\ln \mu(\sigma, F)}{y(\sigma)-\sigma} \leq \bar{\Phi}^{-1}(\sigma), \quad \sigma \in\left[\sigma_{2}, A\right) .
$$

Fix an arbitrary $\sigma \in\left[\sigma_{2}, A\right)$. If $\lambda_{v\left(\sigma, F^{\prime}\right)} \leq \bar{\Phi}^{-1}(\sigma)$, then

$$
\frac{\mu\left(\sigma, F^{\prime}\right)}{\mu(\sigma, F)} \leq \bar{\Phi}^{-1}(\sigma)
$$

by the right of inequalities (10). Therefore, we can further assume that $\lambda_{v\left(\sigma, F^{\prime}\right)}>\bar{\Phi}^{-1}(\sigma)$.

For every integer $n \geq 0$ we have

$$
\left|a_{n}\right| e^{\sigma \lambda_{n}}=\left|a_{n}\right| e^{y(\sigma) \lambda_{n}} e^{(\sigma-y(\sigma)) \lambda_{n}} \leq \mu(y(\sigma), F) e^{(\sigma-y(\sigma)) \lambda_{n}} .
$$

This and (17) imply that

$$
\frac{\left|a_{n}\right| e^{\sigma \lambda_{n}}}{\mu(\sigma, F)} \leq e^{(y(\sigma)-\sigma)\left(\bar{\Phi}^{-1}(\sigma)-\lambda_{n}\right)}, \quad n \geq 0
$$

Since $\lambda_{v\left(\sigma, F^{\prime}\right)}>\bar{\Phi}^{-1}(\sigma)$, from (18) it follows that

$$
\frac{\mu\left(\sigma, F^{\prime}\right)}{\mu(\sigma, F)} \leq \sup _{\lambda_{n}>\bar{\Phi}^{-1}(\sigma)} \lambda_{n} e^{(y(\sigma)-\sigma)\left(\bar{\Phi}^{-1}(\sigma)-\lambda_{n}\right)} .
$$

Let us consider the function

$$
h(t)=t e^{(y(\sigma)-\sigma)\left(\bar{\Phi}^{-1}(\sigma)-t\right)}, \quad t \in \mathbb{R} .
$$

It is easy to check that this function is descending on the interval $\left[t_{0},+\infty\right)$, where

$$
t_{0}=\frac{1}{y(\sigma)-\sigma}
$$

Using Lemma 2, we have

$$
t_{0}=\frac{\bar{\Phi}^{-1}(\sigma)}{\Phi(y(\sigma))} \leq \bar{\Phi}^{-1}(\sigma)
$$

and so from (19) it follows that

$$
\frac{\mu\left(\sigma, F^{\prime}\right)}{\mu(\sigma, F)} \leq h\left(\bar{\Phi}^{-1}(\sigma)\right)=\bar{\Phi}^{-1}(\sigma) .
$$

Theorem 3 is proved. 
Proof of Theorem 4. Suppose that $A \in(-\infty,+\infty], \Phi \in \Omega_{A}$, and prove that there exists a Dirichlet series of the form (1) such that for an infinite set $E$ of positive integers we have $a_{n}=e^{-\widetilde{\Phi}\left(\lambda_{n}\right)}$ when $n \in E, a_{n}=0$ when $n \notin E$, and this series satisfies (12).

Let $\varphi(x)=\widetilde{\Phi}_{+}^{\prime}(x)$ for all $x \in \mathbb{R}$, and $x_{0}=\inf \{x>0: \Phi(\varphi(x))>0\}$.

Since, by Lemma $1, \widetilde{\Phi}$ is convex on $\mathbb{R}$, we have

$$
\frac{\widetilde{\Phi}(x)-\widetilde{\Phi}(b)}{x-b} \leq \varphi(x), \quad x>b .
$$

In addition, $\bar{\Phi}$ is increasing on $\left(x_{0},+\infty\right)$. Therefore, if $x>b>x_{0}$, then $\bar{\Phi}(x)>\bar{\Phi}(b)$. This implies that

$$
\bar{\Phi}(x)<\frac{\widetilde{\Phi}(x)-\widetilde{\Phi}(b)}{x-b}, \quad x>b>x_{0} .
$$

Now we show that

$$
\bar{\Phi}(x)-\bar{\Phi}(b)=o(\Phi(\varphi(x))), \quad x \rightarrow+\infty .
$$

Since, by Lemma $1, \bar{\Phi}(x) \rightarrow A$ and $\Phi(\varphi(x)) \rightarrow+\infty$ as $x \rightarrow+\infty$, relation (22) is obvious in the case $A<+\infty$. If $A=+\infty$, then we get

$$
\bar{\Phi}(x)<\varphi(x)=o(\Phi(\varphi(x)))
$$

as $x \rightarrow+\infty$, and this also implies (22).

It follows from the above that there exists a sequence $\left(n_{k}\right)$ of positive integers such that we have $\lambda_{n_{0}}>x_{0}$ and also

$$
\begin{gathered}
\lambda_{n_{k}}=o\left(\lambda_{n_{k+1}}\right), \quad k \rightarrow \infty \\
\bar{\Phi}\left(\lambda_{n_{k+1}}\right)>\varphi\left(\lambda_{n_{k}}\right), \quad k \geq 0 ; \\
\lambda_{n_{k}}\left(\bar{\Phi}\left(\lambda_{n_{k+1}}\right)-\bar{\Phi}\left(\lambda_{n_{k}}\right)\right)=o\left(\Phi\left(\varphi\left(\lambda_{n_{k+1}}\right)\right)\right), \quad k \rightarrow \infty .
\end{gathered}
$$

For each $k \geq 0$ we set

$$
\sigma_{k}=\bar{\Phi}\left(\lambda_{n_{k+1}}\right), \quad \varkappa_{k}=\frac{\widetilde{\Phi}\left(\lambda_{n_{k+1}}\right)-\widetilde{\Phi}\left(\lambda_{n_{k}}\right)}{\lambda_{n_{k+1}}-\lambda_{n_{k}}} .
$$

Using (21) and (20) with $x=\lambda_{n_{k+1}}$ and $b=\lambda_{n_{k}}$, as well as (24), we obtain

$$
\sigma_{k}=\bar{\Phi}\left(\lambda_{n_{k+1}}\right)<\varkappa_{k} \leq \varphi\left(\lambda_{n_{k+1}}\right)<\bar{\Phi}\left(\lambda_{n_{k+2}}\right)=\sigma_{k+1}, \quad k \geq 0 .
$$

This implies that $\left(\varkappa_{k}\right)$ is a sequence increasing to $A$.

Let $\sigma_{0}=\bar{\Phi}\left(x_{0}+0\right)$, and $y(\sigma)=\varphi\left(\bar{\Phi}^{-1}(\sigma)\right)$ for all $\sigma \in\left(\sigma_{0}, A\right)$. Using (23) and (25), we have

$$
\varkappa_{k}=\bar{\Phi}\left(\lambda_{n_{k+1}}\right)+\frac{\lambda_{n_{k}}\left(\bar{\Phi}\left(\lambda_{n_{k+1}}\right)-\bar{\Phi}\left(\lambda_{n_{k}}\right)\right)}{\lambda_{n_{k+1}}-\lambda_{n_{k}}}=\sigma_{k}+\frac{o\left(\Phi\left(\varphi\left(\lambda_{n_{k+1}}\right)\right)\right)}{\lambda_{n_{k+1}}}=\sigma_{k}+\frac{o\left(\Phi\left(y\left(\sigma_{k}\right)\right)\right)}{\bar{\Phi}^{-1}\left(\sigma_{k}\right)}
$$

as $k \rightarrow \infty$. From this and from Lemma 3 we see that

$$
\bar{\Phi}^{-1}\left(\varkappa_{k}\right) \sim \bar{\Phi}^{-1}\left(\sigma_{k}\right), \quad k \rightarrow \infty
$$

Put $a_{n_{k}}=e^{-\widetilde{\Phi}\left(\lambda_{n_{k}}\right)}$ for all $k \geq 0$, and let $a_{n}=0$ if $n \neq n_{k}$ for every $k \geq 0$, i.e., $E=\left\{n_{0}, n_{1}, \ldots\right\}$. Consider series (1) with such coefficients $a_{n}$. Since

$$
\varkappa_{k}=\frac{\ln a_{n_{k}}-\ln a_{n_{k+1}}}{\lambda_{n_{k+1}}-\lambda_{n_{k}}} \uparrow A, \quad k \rightarrow \infty,
$$


for this series by Lemma 4 we have $\lambda_{v\left(\varkappa_{k}, F\right)}=\lambda_{n_{k+1}}, k \geq 0$. Therefore, using (26), we get

$$
\varlimsup_{\sigma \uparrow A} \frac{\lambda_{v(\sigma, F)}}{\bar{\Phi}^{-1}(\sigma)} \geq \varlimsup_{k \rightarrow \infty} \frac{\lambda_{v\left(\varkappa_{k}, F\right)}}{\bar{\Phi}-1\left(\varkappa_{k}\right)}=\varlimsup_{k \rightarrow \infty} \frac{\lambda_{n_{k+1}}}{\bar{\Phi}^{-1}\left(\sigma_{k}\right)}=1 .
$$

Theorem 4 is proved.

Proof of Theorem 1. Let $A \in(-\infty,+\infty]$ and $\Phi \in \Omega_{A}$.

(i) Suppose that a Dirichlet series of the form (1) with $\beta(F)=A$ satisfies the condition $t_{\Phi}(F) \leq 1$. Let $q>1$ be an arbitrary fixed number, and let $\Psi(\sigma)=q \Phi(\sigma)$ for all $\sigma \in D_{\Phi}$. Then, as it is easy to see, $\bar{\Psi}^{-1}(\sigma)=q \bar{\Phi}^{-1}(\sigma)$ for each $\sigma \in\left(A_{0}, A\right)$. From the condition $t_{\Phi}(F) \leq 1$ it follows that $\ln \mu(\sigma, F) \leq \Psi(\sigma), \sigma \in\left[\sigma_{1}, A\right)$. Therefore, by Theorem 3 we have

$$
\frac{\mu\left(\sigma, F^{\prime}\right)}{\mu(\sigma, F)} \leq \bar{\Psi}^{-1}(\sigma)=q \bar{\Phi}^{-1}(\sigma), \quad \sigma \in\left[\sigma_{2}, A\right) .
$$

Since $q>1$ is arbitrary, this implies estimate (7).

(ii) By Theorem 4 there exists a Dirichlet series of the form (1) such that for an infinite set $E$ of nonnegative integers we have $a_{n}=e^{-\widetilde{\Phi}\left(\lambda_{n}\right)}$ if $n \in E$ and $a_{n}=0$ if $n \notin E$, and this series satisfies (12). Then $\beta(F)=A$ (see Remark 4). Using (13), for this series we obtain $t_{\Phi}(F)=1$, and hence, by the first part of our theorem, we have (7). From (7) and (12), due to the left of inequalities (10), we immediately obtain (9).

Theorem 1 is proved.

Proof of Theorem 2. Suppose that $A \in(-\infty,+\infty], \Phi \in \Omega_{A}$, and $\Gamma$ is defined by (14). First, let us prove that there exists a function $\Theta \in \Omega_{A}$ such that $\bar{\Theta}(x)=\Gamma(x)$ for all $x \in[\alpha,+\infty)$.

Let $\varphi(x)=\widetilde{\Phi}_{+}^{\prime}(x), x \in \mathbb{R}$. Put

$$
\theta(x)=\varphi(x)-\frac{1}{x}, \quad x \in[\alpha,+\infty) .
$$

Since $\alpha>e$ (see above), the function $\theta$ is increasing and continuous from the right on $[\alpha,+\infty)$, and also $\lim _{x \uparrow+\infty} \theta(x)=A$. Consider the function

$$
\theta^{-1}(\sigma)=\inf \{x \in[\alpha,+\infty): \theta(x)>\sigma\}, \quad \sigma \in[a, A),
$$

where $a=\theta(\alpha)$. By Lemma 5 , the function $\theta^{-1}$ is nondecreasing continuous on $[a, A)$, and also

$$
\theta(x)=\max \left\{\sigma \in[a, A): \theta^{-1}(\sigma) \leq x\right\}, \quad x \in[\alpha,+\infty) .
$$

Put

$$
\Theta_{0}(\sigma)=\int_{a}^{\sigma} \theta^{-1}(t) d t, \quad \sigma \in[a, A) .
$$

Let $A<+\infty$, and let $\eta(x)=A-\frac{1}{x}, x \in[\alpha,+\infty)$. Since $\varphi(x)<A$ for all $x \in \mathbb{R}$, we have $\theta(x)<\eta(x)$ for each $x \in[\alpha,+\infty)$. Then

$$
\theta^{-1}(\sigma) \geq \eta^{-1}(\sigma)=\frac{1}{A-\sigma^{\prime}}, \quad \sigma \in[a, A),
$$

and hence for all $\in[a, A)$ we get

$$
\Theta_{0}(\sigma) \geq \int_{a}^{\sigma} \frac{d t}{A-\sigma}=\ln \frac{A-a}{A-\sigma} .
$$


This implies that $\Theta_{0}(\sigma) \rightarrow+\infty$ as $\sigma \rightarrow A-0$. In the case $A=+\infty$, for all sufficiently large $\sigma$ we have

$$
\Theta_{0}(\sigma) \geq \int_{\sigma / 2}^{\sigma} \theta^{-1}(t) d t \geq \frac{\sigma}{2} \theta^{-1}\left(\frac{\sigma}{2}\right)
$$

This implies that $\Theta_{0}(\sigma) / \sigma \rightarrow+\infty$ as $\sigma \rightarrow \infty$. Therefore, $\Theta_{0} \in \Omega_{A}$ always.

Let $x \in[\alpha,+\infty)$ be an arbitrary fixed number. Consider the function

$$
h(\sigma)=x \sigma-\Theta_{0}(\sigma), \quad \sigma \in[a, A) .
$$

Since $h^{\prime}(\sigma)=x-\theta^{-1}(\sigma)$, the function $h$ assumes its maximum value on $[a, A)$ at the point $\sigma=\theta(x)$, and this point is maximal among all possible maximum points of $h$.

Therefore, from Lemma 1 we can see that for all $x \in[\alpha,+\infty)$ the function $\theta(x)$ is defined by $\Theta$ as well as $\varphi(x)$ by $\Phi$, and hence $\theta(x)=\widetilde{\Theta}_{+}^{\prime}(x)$. Put $C=-\widetilde{\Phi}(\alpha)+\ln \alpha+\widetilde{\Theta}(\alpha)$ and let $\Theta(\sigma)=\Theta_{0}(\sigma)+C$ for all $\sigma \in[a, A)$. Then $\Theta \in \Omega_{A}$ and for every $x \in[\alpha,+\infty)$ we have

$$
\begin{aligned}
\widetilde{\Theta}(x)=\widetilde{\Theta}_{0}(x)-C & =\int_{\alpha}^{x} \theta(t) d t+\widetilde{\Theta}_{0}(\alpha)-C=\int_{\alpha}^{x}\left(\varphi(t)-\frac{1}{t}\right) d t+\widetilde{\Theta}_{0}(\alpha)-C \\
& =\widetilde{\Phi}(x)-\ln x-\widetilde{\Phi}(\alpha)+\ln \alpha+\widetilde{\Theta}_{0}(\alpha)-C=\widetilde{\Phi}(x)-\ln x=x \Gamma(x),
\end{aligned}
$$

and hence $\bar{\Theta}(x)=\Gamma(x)$.

(i) Suppose that a Dirichlet series of the form (1) with $\beta(F)=A$ satisfies the condition $t_{\Phi}(F) \leq 1$. Let $q>1$ be an arbitrary fixed number. Then $t_{\Phi}(F)<q$, and therefore from (13) for all $n \geq n_{1}$ we obtain the inequality

$$
\lambda_{n} \leq q \bar{\Phi}^{-1}\left(\frac{1}{\lambda_{n}} \ln \frac{1}{\left|a_{n}\right|}\right)
$$

which, as is easy to see, is equivalent to the inequality

$$
\ln \left|a_{n}\right| \leq-q \widetilde{\Phi}\left(\frac{\lambda_{n}}{q}\right)
$$

Hence, for all $n \geq n_{2}$ we have

$$
\ln \left|\lambda_{n} a_{n}\right| \leq \ln \lambda_{n}-q \widetilde{\Phi}\left(\frac{\lambda_{n}}{q}\right)=-q \widetilde{\Theta}\left(\frac{\lambda_{n}}{q}\right)+(1-q) \ln \lambda_{n}+q \ln q \leq-q \widetilde{\Theta}\left(\frac{\lambda_{n}}{q}\right),
$$

which implies that

$$
\lambda_{n} \leq q \bar{\Theta}^{-1}\left(\frac{1}{\lambda_{n}} \ln \frac{1}{\left|\lambda_{n} a_{n}\right|}\right) .
$$

Therefore, using (13) with $F^{\prime}$ and $\Theta$ instead of $F$ and $\Phi$ respectively, we obtain $t_{\Theta}\left(F^{\prime}\right) \leq q$. Since $q>1$ is arbitrary, this implies that $t_{\Theta}\left(F^{\prime}\right) \leq 1$.

Recalling that $\bar{\Theta}(x)=\Gamma(x)$ for all $x \in[\alpha,+\infty)$, and using Theorem 1 with $F^{\prime}$ and $\Theta$ instead of $F$ and $\Phi$ respectively, we have

$$
\varlimsup_{\sigma \uparrow A} \frac{\lambda_{v\left(\sigma, F^{\prime}\right)}}{\Gamma^{-1}(\sigma)}=\varlimsup_{\sigma \uparrow A} \frac{\lambda_{v\left(\sigma, F^{\prime}\right)}}{\bar{\Theta}-1(\sigma)} \leq \varlimsup_{\sigma \uparrow A} \frac{\mu\left(\sigma, F^{\prime \prime}\right)}{\mu\left(\sigma, F^{\prime}\right) \bar{\Theta}^{-1}(\sigma)} \leq 1 .
$$

(ii) Since $\Theta \in \Omega_{A}$, by Theorem 4 there exists a Dirichlet series of the form

$$
G(s)=\sum_{n=0}^{\infty} b_{n} e^{s \lambda_{n}}
$$


such that for an infinite set $E$ of positive integers we have $b_{n}=e^{-\widetilde{\Theta}\left(\lambda_{n}\right)}$ if $n \in E$ and $b_{n}=0$ if $n \notin E$, and this series satisfies the equation

$$
\varlimsup_{\sigma \uparrow A} \frac{\lambda_{v(\sigma, G)}}{\bar{\Theta}-1(\sigma)}=1
$$

We note also that $\beta(G)=A$ (see Remark 4).

Put $a_{n}=e^{-\widetilde{\Phi}\left(\lambda_{n}\right)}=b_{n} / \lambda_{n}$ if $n \in E$ and $a_{n}=0$ if $n \notin E$, and consider series (1) with such coefficients $a_{n}$. For this series we have $F^{\prime}=G$, and hence $\beta(F)=\beta(G)=A$. By (13) we obtain $t_{\Phi}(F)=1$. In addition, for this series equality (15) holds, because this equality coincides with (27). Theorem 2 is proved.

\section{REFERENCES}

[1] Evgrafov M.A. Asymptotic estimates and entire functions. Nauka, Moscow, 1979. (in Russian)

[2] Fedynyak S.I., Filevych P.V. Distance between a maximum modulus point and zero set of an analytic function. Mat. Stud. 2019, 52 (1), 10-23. doi:10.30970/ms.52.1.10-23

[3] Fedynyak S.I., Filevych P.V. Growth estimates for a Dirichlet series and its derivative. Mat. Stud. 2020, 53 (1), 3-12. doi:10.30970/ms.53.1.3-12

[4] Filevych P.V. On relations between the abscissa of convergence and the abscissa of absolute convergence of random Dirichlet series. Mat. Stud. 2003, 20 (1), 33-39.

[5] Filevych P.V., Hrybel O.B. The growth of the maximal term of Dirichlet series. Carpathian Math. Publ. 2018, 10 (1), 79-81. doi:10.15330/cmp.10.1.79-81

[6] Filevich P.V. On Valiron's theorem on the relations between the maximum modulus and the maximal term of an entire Dirichlet series. Russian Math. 2004, 48 (4), 63-69. (translation of Izv. Vyssh. Uchebn. Zaved. Mat. 2004, (4), 66-72. (in Russian))

[7] Hlova T.Ya., Filevych P.V. Generalized types of the growth of Dirichlet series. Carpathian Math. Publ. 2015, 7 (2), 172-187. doi:10.15330/cmp.7.2.172-187

[8] Leont'ev A.F. Series of exponents. Nauka, Moscow, 1976. (in Russian)

[9] Sheremeta M.N. On the maximum term of the derivative of the Dirichlet series. Russian Math. 1998, 42 (5), 66-70. (translation of Izv. Vyssh. Uchebn. Zaved. Mat. 1998, (5), 68-72. (in Russian))

Received 21.04.2020

Фединяк С.І., Філевич П.В. Оиінки зростання максимального илена та иентрального показника похідної ряду Аіріхле // Карпатські матем. публ. — 2020. — Т.12, №2. - C. 269-279.

Нехай $A \in(-\infty,+\infty], \Phi:[a, A) \rightarrow \mathbb{R}-$ довільна неперервна функція така, що $x \sigma-\Phi(\sigma) \rightarrow$ $-\infty, \sigma \uparrow A$, Аля кожного $x \in \mathbb{R}, \widetilde{\Phi}(x)=\max \{x \sigma-\Phi(\sigma): \sigma \in[a, A)\}-$ функція, спряжена 3 $\Phi$ за Юнгом, $\bar{\Phi}(x)=\widetilde{\Phi}(x) / x$ і $\Gamma(x)=(\widetilde{\Phi}(x)-\ln x) / x$ Аля всіх достатньо великих $x,\left(\lambda_{n}\right)$ невід'ємна зростаюча до $+\infty$ послідовність, а $F(s)=\sum_{n=0}^{\infty} a_{n} e^{\text {s } \lambda_{n}}-$ ряд $\Delta$ іріхле, максимальний член $\mu(\sigma, F)=\max \left\{\left|a_{n}\right| e^{\sigma \lambda_{n}}: n \geq 0\right\}$ та центральний індекс $v(\sigma, F)=\max \left\{n \geq 0:\left|a_{n}\right| e^{\sigma \lambda_{n}}=\right.$ $\mu(\sigma, F)\}$ якого визначені для всіх $\sigma<A$. Аоведено, що якщо $\ln \mu(\sigma, F) \leq(1+o(1)) \Phi(\sigma), \sigma \uparrow A$, то виконуються нерівності

$$
\varlimsup_{\sigma \uparrow A} \frac{\mu\left(\sigma, F^{\prime}\right)}{\mu(\sigma, F) \bar{\Phi}^{-1}(\sigma)} \leq 1, \quad \varlimsup_{\sigma \uparrow A} \frac{\lambda_{\nu\left(\sigma, F^{\prime}\right)}}{\Gamma^{-1}(\sigma)} \leq 1,
$$

і ці нерівності є точними.

Ключові слова і фрази: ряд Аіріхле, максимальний член, центральний індекс, центральний показник, спряжена за Юнгом функція. 